\title{
Pour ne plus voir d'émeutes de la faim : une troisième frontière agraire à explorer?
}

Voici qu'au début du XXI e siècle, il y a de nouveau des pays entiers où l'on a peur de mourir de faim! Tout indiquait que les engagements des Sommets mondiaux de l'alimentation de la FAO de réduire la faim dans le monde d'ici 2015, au nom du droit de l'homme à se nourrir, ne seraient pas tenus et que les quelque 800 millions de ruraux qui souffrent d'une sous-alimentation calorique chronique risquaient bien d'être encore plus nombreux à l'issue de la décennie à venir. Mais que des masses urbaines soient menacées de famine, parce qu'elles ne peuvent plus acheter leur nourriture, alors que cette année - hormis en Australie, victime d'une sécheresse récurrente -, les récoltes sont plutôt bonnes, paraît surprenant et inquiétant. De fait, les prix des denrées agricoles ont augmenté considérablement en une année, avec des accélérations impressionnantes ces derniers mois : de $130 \%$ pour le blé, de $70 \%$ pour le maïs et de 50 à $120 \%$ pour le riz selon les qualités. C'est que les céréales sont devenues des biens rares et coûteux : augmentation des coûts de l'énergie pour les produire et les transporter, spéculation sur les stocks, concurrence avec les incitations des pays industrialisés à les utiliser comme biocarburants et conséquences des taux de croissance «à deux chiffres » des pays dits émergents, devant lesquels tout le monde rêve. Comme si nous ne savions pas que lorsqu'on est moins pauvre... on mange plus et mieux! Certes, la faim n'affecte pas tout le monde, mais elle sévit suffisamment pour que soient bousculés les équilibres planétaires de produits agricoles dont la part mise en marché reste réduite (moins de $10 \%$ pour le riz, $4 \%$ cette année). Ainsi, tout d'un coup, la quantité importée chaque année par les pays africains pour leur survie ( $40 \%$ de leur consommation, ce qui représente une part considérable pour eux mais un pourcentage minime du marché mondial) s'est reportée vers d'autres marchés pour satisfaire ces nouveaux besoins solvables ou même tout simplement du fait de la décision de quelques exportateurs mondiaux (de riz en particulier, dont on ne tire pourtant pas encore de bioéthanol)... de ne plus exporter afin d'assurer leur propre garde-manger! Assis sur leurs certitudes que les stocks resteraient importants (il n'y en a plus!), que les prix des biens alimentaires resteraient bas pour des années (ils ont explosé !), les meilleurs experts mondiaux conseillaient aux pays considérés comme peu compétitifs en denrées alimentaires de se spécialiser dans quelques productions « exotiques » destinées à l'exportation vers les pays occidentaux et, en conséquence, d'importer l'essentiel de leur propre nourriture. Les silos attendent dans les ports! Par exemple, les pays de la rive sud de la Méditerranée, qui représentent $4 \%$ de la population mondiale (dont $64 \%$ dans les villes qui congestionnent le littoral), captent $12 \%$ des importations mondiales de céréales...

Alors voilà qu'on s'intéresse de nouveau à l'agriculture comme une priorité mondiale. La Banque du même nom, qui ne s'était pas préoccupée officiellement de cette question depuis 27 ans, vient de consacrer son rapport annuel 2008 à l'agriculture pour rappeler que c'est là une activité utile, génératrice de croissance, qui peut probablement constituer un des moyens de lutte les plus efficaces pour résorber la pauvreté rurale et pour maintenir des activités (et donc des infrastructures et des services) dans les campagnes et les brousses. De plus, par son emprise sur les terres qu'elle partage avec la forêt, l'agriculture constitue un des facteurs essentiels de la préservation de l'environnement (sols, eau, biodiversité...) et de la plupart des services des écosystèmes continentaux à l'échelle de la planète. L'International Assessment of Agricultural Science \& Technology for Development (IAASTD, conçu par les Nations unies dans l'esprit du GIEC et du Millennium Ecosystem Assessment, et que nous avons déjà évoqué dans ces pages) vient, à la mi-avril de cette année, de boucler son premier cycle par l'adoption, à Johannesburg, de ses rapports finaux par 60 pays sur les 63 représentés. Les options pour la recherche au niveau mondial sont ainsi posées, avec l'idée forte que les avancées techniques ne suffiront pas si elles ne prennent pas soin de mieux impliquer la diversité 
des acteurs concernés, de reconnaître leurs savoirs et leurs droits, de respecter leurs modes d'organisation sociale, de prendre en compte les enjeux environnementaux... Car non seulement il faut produire plus, mais il faut également produire mieux, tout en s'adaptant au changement climatique! Révolution doublement verte, écoagriculture, agroécologie, agriculture de conservation s'affichent en première page de l'agenda!

La question de la frontière agraire, qui paraissait un peu oubliée, revient à l'ordre du jour, avec toutefois une troisième dimension du fait de cette nouvelle situation. À la première frontière, bien connue depuis le Néolithique, celle de la défriche et de la mise en culture des « terres vierges ", s'en est inexorablement ajoutée une deuxième depuis un peu plus d'un siècle, celle du développement urbain et des infrastructures. Là, les réglementations et le marché foncier font la loi, et il est bien rare que la valorisation agricole puisse faire front aux autres spéculations ou décisions d'intérêt général. Les défenseurs de l'intangibilité de la première frontière le font de nos jours au nom des enjeux environnementaux. Les questions de biodiversité, de protection des sols, de préservation des ressources en eau et de leur qualité, le ralentissement du changement climatique par la fixation du carbone justifient en effet que l'on arrête de détruire les grandes forêts de la planète (Amazonie, bassin du Congo...) et de drainer les zones humides pour leur «mise en valeur agricole », sauf éventuellement compensation comme aux États-Unis. Il faut donc alors intensifier sans toucher à la frontière et développer si possible une agriculture plus " propre » sur les espaces qui lui sont dédiés, à l'image de l'écoagriculture de Jeff McNeely, le directeur scientifique del'UICN. De ce point de vue, on peut même se demander si les services des écosystèmes, tant vantés aujourd'hui, ne sont pas destinés à assurer une compensation et une garantie vis-à-vis de quelques grands équilibres planétaires, dans l'hypothèse de dérapages techniques divers (contaminants biologiques et abiotiques), face au défi technologique que représente le fait de nourrir, à l'horizon de 2050, 9 milliards d'habitants. Même s'il ne s'agit pas aujourd'hui de présenter cette frontière comme une réserve foncière, la question se posera de toute évidence pour la taïga, quand la disparition du permafrost attisera les convoitises pour la «mise en valeur» de ces sols regorgeant d'une matière organique stockée depuis les dernières glaciations et qui ne demande qu'à libérer $\mathrm{du}$ $\mathrm{CO}_{2}$ : quelques belles années avant leur épuisement!
N'est-il pas temps de poser la question autrement et $\mathrm{d}$ 'instruire une troisième frontière : celle qui supposerait de revisiter les conceptions mêmes et les pratiques de l'activité agricole, de penser autrement cette tripartition du monde entre la ville où se concentre la grande majorité de la population, l'espace rural dédié à la production agricole ou forestière et les espaces naturels garants d'une offre de nature comme de la préservation des grands équilibres planétaires? Certes, il s'agira bien de concevoir une diversité et une complémentarité de formes d'agriculture reposant sur des savoirs et des pratiques, des modèles techniques et des circuits de commercialisation qui se singulariseront selon les productions, les situations géographiques, les formes d'action publique, les valeurs culturelles, les engagements sur un mode individuel ou collectif. De telles agricultures pourraient mailler les espaces urbains, pénétrer les lisières forestières pour y introduire des mosaïques écologiques inédites, inventer des façons de produire davantage inspirés de l'écologie que de la chimie, sur les modèles de l'agroécologie d'Altieri. De telles agricultures réhabiliteraient la diversité et la variabilité du matériel génétique animal et végétal, et sauraient s'accommoder des pentes, des bas-fonds, des lisières, des étages et des strates boisés, pour les respecter tout en produisant... et en se reproduisant. Toutefois, il ne s'agit plus seulement de respecter l'environnement ou, pire, de concilier l'agriculture et l'environnement, mais de faire en sorte que l'environnement soit bien, parmi d'autres, une des productions de l'agriculture!

Pour cela, il est donc nécessaire de sortir d'un monde du cloisonnement où espaces, activités, productions, savoirs et recherche obéissent à la même loi de la spécialisation. NSS - au risque de passer aux yeux de certains pour des cahiers d'une agronomie obsolète - a déjà pris l'initiative de rendre compte d'expériences de pratiques agricoles, forestières ou aquacoles innovantes, préoccupées justement de tracer cette nouvelle frontière. Mais cela ne devrait plus être anecdotique, car cette nouvelle frontière est devant nous, sur l'ensemble de la planète, et certainement plus facile à énoncer ici qu'à mettre en œuvre Mais n'est-ce pas là une utopie que la recherche peut transformer en réalité, si elle se donne les moyens de combiner et conjuguer ses savoirs sur les hommes, les sociétés et leurs institutions, sur les milieux, les ressources et les dynamiques qui les animent?

Bernard Hubert Jean-Paul Billaud 